\title{
Biotechnological Innovations through Fungi
}

\author{
Gupta VK ${ }^{1}$, Grigoriev IV ${ }^{2}$, Berrin $\mathrm{JG}^{3}$, Upadhyay $\mathrm{RS}^{4}$, Zeilinger-Migsich $\mathrm{S}^{5}$ \\ ${ }^{1}$ Molecular Glyco-biotechnology Group, Discipline of Biochemistry, National University of Ireland Galway, \\ Ireland Phone: +353862001820 \\ ${ }^{2}$ Department of Energy Joint Genome Institute, 2800 Mitchell Drive, Walnut Creek, The Center for Integrative \\ Genomics, University of California and Genomics Division, Lawrence Berkeley National Laboratory, Mailstop \\ 84-171, Berkeley, CA 94720, USA \\ ${ }^{3}$ Laboratoire de Biotechnologie des Champignons Filamenteux, Marseille, France \\ ${ }^{4}$ Department of Botany, Dy. Coordinator, Centre of Advanced Study, Banaras Hindu University Varanasi 221 \\ 005, India. \\ ${ }^{5}$ Institute of Microbiology, University of Innsbruck, Technikerstrasse 25, 6020 Innsbruck, Austria
}

Email:vijaifzd@gmail.com; vijai.gupta@nuigalway.ie

Gupta VK, Grigoriev IV, Berrin JG, Upadhyay RS, Zeilinger-Migsich S 2016 Biotechnological Innovations through Fungi. Mycosphere 7 (10), 1490, Doi 10.5943/mycosphere/si/3b/11

Research on fungal systems assumes a key part in the biotechnological and biomedical sectors. It has transformed into a subject of growing importance as new fungi and their related biomolecules are described. Fungal interactions with their biotic and abiotic environment is essential to various procedures occurring in the biosphere. The indigenous habitats and hosts of these eukaryotic microorganisms are to a great degree, variously reflected, by the way that fungi are far reaching and found in almost every organic group on Earth. This metabolic flexibility makes fungus intriguing organisms for a scope of industrially important biotechnological applications. Understanding the science of particular fungi in contrasting natural biological communities and their relationship with their living and non-living surroundings is vital to bolster effective and innovative enhancements. To comprehend the potential and to truly grasp the different qualities and science of these eukaryotes, continuing with change of experimental design and strategies are significant. The basic target of this volume is to compile the work from the experts in various researches of fungal biology and biotechnology and review the most recent advances using current developments. 